\title{
Increased osteogenesis with hydroxyapatite constructs combined with serially-passaged bone marrow-derived mesenchymal stem cells
}

\author{
Manabu Akahane ${ }^{1 *}$, Tomoyuki Ueha ${ }^{2}$, Takamasa Shimizu ${ }^{2}$, Yusuke Inagaki $^{2}$, Akira Kido $^{2}$, \\ Tomoaki Imamura ${ }^{1}$, Kenji Kawate ${ }^{3}$, Yasuhito Tanaka ${ }^{2}$ \\ ${ }^{1}$ Department of Public Health, Health Management and Policy, Nara Medical University School of Medicine, Nara, Japan; \\ *Corresponding Author: makahane@naramed-u.ac.jp \\ ${ }^{2}$ Department of Orthopedic Surgery, Nara Medical University, Nara, Japan \\ ${ }^{3}$ Department of Artificial Joint and Regenerative Medicine, Nara Medical University, Nara, Japan
}

Received 18 June 2012; revised 16 July 2012; accepted 17 August 2012

\section{ABSTRACT}

We have previously reported on both the osteogenic potential of hydroxyapatite (HA) combined with bone marrow-derived mesenchymal stem cells (BMSCs) and a method involving osteogenic matrix cell sheet transplantation of BMSCs. In the present study, we assessed the osteogenic potential of serially-passaged BMSCs, both in vitro and in vivo. We also assessed whether an additional cell-loading technique can regain the osteogenic potential of the constructs combined with serially-passaged BMSCs. The present study revealed that passage (P) 1 cells cultured in osteogenic-induced medium showed strong positive staining for alkaline phosphatase (ALP) and Alizarin Red S, whereas P3 cells showed faint staining for ALP, with no Alizarin Red S staining. Staining of P1, P2 and P3 cells were progressively weaker, indicating that the osteogenic potential of the serially-passaged rat BMSCs is lost after P3 in vitro. The in vivo study showed that little bone formation was observed in the HA constructs seeded with P3 cells, 4 weeks after subcutaneous implantation. However, P3 cell/HA constructs which had increased cell-loading showed abundant bone formation within the pores of the HA construct. ALP and osteocalcin mRNA expression in these constructs was significantly higher than that of constructs with regular cell-seeding. The present study indicates that the osteogenic potential of the constructs with serially-passaged BMSCs is increased by additional cell-loading. This method can be applied to cases requiring hard tissue reconstruction, where BMSCs require serial expansion of cells.

Keywords: Osteogenesis; Mesenchymal Stem Cells; Serial Passaging; Hydroxyapatite; Tissue Engineering; Bone Reconstruction

\section{INTRODUCTION}

Bone marrow contains a population of undifferentiated cells known as mesenchymal stem cells (MSCs) $[1,2]$. Bone marrow-derived MSCs (BMSCs) have the potential for self-renewal and differentiation into several cell types after culture expansion in vitro and in vivo, including osteoblastic, chondrocytic, adipocytic and neuronal cells [3-7]. Differentiation into osteoblastic lineage cells after subculture in osteogenic-induced media, containing dexamethasone (Dex), ascorbic acid phosphate and $\beta$-glycerophosphate ( $\beta$-GP), has been shown for rat and human BMSCs [8-11]. The osteogenic phenotypic markers, alkaline phosphatase (ALP) and osteocalcin (OC), can be detected by days $10-12$ in passage 1 (P1)-cultured cells, including mineralization [12]. The combination of BMSCs with a scaffolding material, such as hydroxyapatite (HA), can promote the formation of new bone tissue in vivo after subcutaneous transplantation with freshly isolated bone marrow cells [13] or culture-expanded BMSCs (HA/ BMSCs construct) [2,14-17], Recently, we reported a new cell transplantation technique in which BMSCs were cultured in culture medium containing Dex and ascorbic acid phosphate and transplanted as cell sheets [18]. These cell sheets can be combined with HA, in which they resulted in abundant bone formation compared with conventional techniques [17].

Clinical applications of tissue engineered BMSCs have been attempted in a number of therapies, including the treatment of osteonecrosis [19-21]. In these appro- 
aches, BMSCs are obtained from the patients, following which they generally need to be expanded by serial passaging in vitro as the number of cells generated in primary culture is limited. However, the osteogenic potential of BMSCs might be lost by serial passaging in vitro, resulting in decreased osteogenesis after subcutaneous transplantation [8]. Therefore, it is important to determine whether HA constructs combined with seriallypassaged BMSCs maintain sufficient osteogenic potential after in vivo transplantation. In the present study, we used BMSCs obtained from rat bone marrow and assessed the osteogenic potential of serially-passaged BMSCs, both in vitro and in vivo. We also assessed whether an additional cell-loading technique, where cells are cultured in osteoblastic cell sheet preparation medium can regain the osteogenic potential of the constructs combined with serially-passaged BMSCs.

\section{MATERIALS AND METHODS}

\subsection{Bone Marrow Cell Preparation and Cell Culture}

Approval from the animal experimental review board of Nara Medical University was obtained before beginning the experiments. Institutional guidelines for the care and use of laboratory animals have been observed. We previously reported the method of bone marrow cell preparation $[11,18]$. Briefly, bone marrow cells were obtained from the femur shafts of 7-week-old male Fischer 344 rats. Both ends of the femur were removed from the epiphysis and the marrow was flushed out using $10 \mathrm{ml}$ of standard culture medium which was expelled from a syringe containing a 20 -gauge needle. Standard culture medium consisted of minimal essential medium (MEM; Nacalai Tesque, Kyoto, Japan) containing 15\% fetal bovine serum (FBS, JRH Bioscience Inc., KS, USA) and antibiotics $(100 \mathrm{U} / \mathrm{ml}$ penicillin and $100 \mu \mathrm{g} / \mathrm{ml}$ streptomycin, Nacalai Tesque).

The obtained cells were collected in two T-75 flasks (Falcon, BD, NJ, USA) containing $15 \mathrm{ml}$ of standard culture medium. These cells were known as passage 0 (P0). Cell culture was maintained in a humidified atomsphere of $95 \%$ air with $5 \% \mathrm{CO}_{2}$ at $37^{\circ} \mathrm{C}$. After reaching confluency, cells were released from the culture substratum using trypsin/EDTA (Gibco, Invitrogen, CA, USA). The released cells were seeded at density of $1 \times 10^{4}$ cells $/ \mathrm{cm}^{2}$ for subculture in T75 flasks (Falcon) for serial passaging to obtain passage 3 (P3) cells. Each passage of cells (P1, P2 and P3) were used for the following experiments.

\subsection{ALP and Alizarin Red S Staining}

To evaluate the osteogenic potential of serially-passaged cells in vitro, $\mathrm{P} 1, \mathrm{P} 2$ and $\mathrm{P} 3$ cells were seeded in 6-well plates at density of $1 \times 10^{4}$ cells $/ \mathrm{cm}^{2}$ and cultured with or without osteogenic-induced media containing Dex $(10 \mathrm{nM})$, ascorbic acid phosphate $(82 \mu \mathrm{g} / \mathrm{mL})$ and $\beta$-GP $(10 \mathrm{mM})$ for 14 days. Each well was stained with Naphtol-AS-MX phosphate sodium salt (Sigma) and Fast Red Violet B (Nacalai Tesque) for ALP staining. $10 \mathrm{mg}$ of each reagent was dissolved in $20 \mathrm{ml}$ of AMP buffer (1.0 mM $\mathrm{MgCl}_{2}, 10 \mathrm{mM}$ p-nitrophenyl phosphate in 0.056 M 2-amino-2-methyl-propanol). Cells were incubated with $2 \mathrm{ml}$ of the substrate solution at room temperature for $\sim 2$ minutes and rinsed with $\mathrm{dH}_{2} \mathrm{O}$. Alizarin Red S (0.5\%) was also used to evaluate calcium deposition, following which cells were rinsed with $\mathrm{dH}_{2} \mathrm{O}$ $[12,18]$. Each experiment contained 3 replicates and was performed in duplicate.

\subsection{ALP Activity, Calcium and DNA Concentration in Vitro}

Cells cultured in 12-well culture plates were used to measure ALP activity, as previously reported [12]. Briefly, P1, P2 and P3 cells, seeded in 12-well plates at a density of $1 \times 10^{4}$ cells $/ \mathrm{cm}^{2}$ and cultured for 14 days with or without osteogenic-induced media containing Dex, ascorbic acid phosphate and $\beta$-GP, were scraped into $1 \mathrm{ml}$ of $0.05 \mathrm{M}$ sodium phosphate buffer, $2 \mathrm{mM}$ EDTA and 2 $\mathrm{M} \mathrm{NaCl}$, following which they were homogenized and sonicated. To measure the ALP activity of cultured cells, $0.1 \mathrm{ml}$ of the sonicated cell suspension was added to 0.5 $\mathrm{ml}$ of $0.2 \%$ Nonidet P-40 containing $1 \mathrm{mM} \mathrm{MgCl} 2$ and centrifuged at $13,000 \mathrm{rpm}$ for 10 minutes at $4^{\circ} \mathrm{C}$. The supernatant was assayed for ALP activity using $p$-nitrophenylphosphate as a substrate. An aliquot $(10 \mu \mathrm{l})$ of the supernatant was added to $1 \mathrm{ml}$ of $50 \mathrm{mM} p$-nitrophenylphosphate containing $1 \mathrm{mM} \mathrm{MgCl}$ and the mixture was incubated for 30 minutes at $37^{\circ} \mathrm{C}$. Subsequently, $2 \mathrm{ml}$ of $0.2 \mathrm{~N} \mathrm{NaOH}$ was added to stop the enzymatic reaction and the absorption at $410 \mathrm{~nm}$ was measured by spectrophotometry. ALP activity was represented as $p$-nitrophenol release $(\mu \mathrm{mol})$ after 30 minutes of incubation at $37^{\circ} \mathrm{C}$.

Calcium was extracted from the sediment of Nonidet P-40 extract with $500 \mu 1$ of $20 \%$ formic acid for 24 hours at $4^{\circ} \mathrm{C}$. An aliquot of the formic acid extract was diluted with strontium solution. Calcium concentration was determined using an atomic absorption spectrometer (AA-610 S; Shimadzu Co., Kyoto, Japan). The sonicated cell suspension was also used for DNA content measurement by fluorescence emission at $458 \mathrm{~nm}$ in the presence of $0.5 \mu \mathrm{g} / \mathrm{ml}$ Hoechst 33,258 (Wako Pure Chemical), with calf thymus DNA as the standard (Wako Pure Chemical). Each group comprised of 4 wells. The experiment was performed in duplicate. 


\subsection{Preparation of HA Constructs Seeded with P3 Cells}

We have previously reported the preparation of HA constructs combined with BMSCs (BMSC/HA construct) $[12,17]$. Briefly, after release from the substratum, P3 cells were centrifuged and resuspended at a density of $1 \times 10^{6} \mathrm{cells} / \mathrm{ml}$ in standard medium. Air was removed from the HA, which were previously soaked in cell suspension, by vacuum prior to making the constructs. Then, the HA disks were soaked in the cell suspension for 2 hours in a $\mathrm{CO}_{2}$ incubator at $37^{\circ} \mathrm{C}$ to create the $\mathrm{P} 3$ cell/ $\mathrm{HA}$ constructs. After soaking in the cell suspension, the construct was transferred into a 12-well plate (Falcon) in 1 $\mathrm{mL}$ of osteogenic-induced medium, consisting of standard medium, $82 \mu \mathrm{g} / \mathrm{mL}$ ascorbic acid phosphate (Wako Pure Chemical Industrials, Kyoto, Japan) and $10 \mathrm{nM}$ Dex (Sigma, St. Louis, MO, USA) for further subculture. After 14 days of subculture, the cultured P3 cell/HA constructs were transplanted into a subcutaneous site of syngenic rats for the control group of the in vivo experiments.

Porous HA ceramics $(50 \%$ average void volume, 5 $\mathrm{mm}$ in diameter and $2 \mathrm{~mm}$ thickness) were used in this study (Cellyard HA scaffold, Pentax Co., Tokyo, Japan). The solid and porous components of the microstructure are interconnected.

\subsection{Additional Cell-Loading of the $\mathrm{P} 3$ Cell/HA Constructs}

We previously reported that BMSCs cultured in standard medium containing Dex and ascorbic acid phosphate resulted in the formation of cell sheets $[10,18]$. In the present study, additional P3 cells for loading were also prepared in cell sheet culture medium. Briefly, P3 cells were seeded at a density of $1 \times 10^{4} \mathrm{cells} / \mathrm{cm}^{2}$ in 3.5 $\mathrm{cm}$ and $10 \mathrm{~cm}$ dishes (Falcon) and cultured in standard medium containing Dex $(10 \mathrm{nM})$ and ascorbic acid phosphate $(82 \mu \mathrm{g} / \mathrm{mL})$ (cell sheet preparation medium) until confluent. We used a mechanical retrieval technique for the preparation of additional P3 cells for cell loading of the constructs. The cells were rinsed twice with phosphate-buffered saline (PBS; Gibco) and lifted as sheet structure using a cell-scraper. As P3 cells formed incomeplete cell sheets compared with P1 cells, they were loaded onto $\mathrm{P} 3$ cell/HA constructs by a short centrifugation at $900 \mathrm{rpm}$ for 2 min (Kubota 5100, Kubota Corp., Tokyo, Japan). Subsequently, the constructs were transplanted into a subcutaneous site as the additional-loading group. Because we used a number of different cell densities for the additional loading of the constructs, we placed them into two subgroups, according to whether they were cultured in $3.5 \mathrm{~cm}$ or $10 \mathrm{~cm}$ dishes.

Thus, there were three groups in the in vivo study: the
P3 cell/HA construct without additional loading (control group), the $\mathrm{P} 3$ cell/HA construct cultured in a $3.5 \mathrm{~cm}$ dish of $\mathrm{P} 3$ cells (small loading group) and the $\mathrm{P} 3$ cell/HA construct cultured in a $10 \mathrm{~cm}$ dish of $\mathrm{P} 3$ cells (large loading group). Each group contained seven HA disks in two recipient rats (three or four disks per rat), which were implanted at subcutaneous sites on their backs. Three disks were used for histology and four for realtime PCR analysis. The experiment was performed in duplicate.

\subsection{Sample Harvest and Histological Analysis}

Implanted disks were harvested 4 weeks after implantation. HA disks for analysis by real-time PCR were frozen until RNA isolation. Disks for histological evaluation were fixed in $10 \%$ buffered formalin after trimming off the excess rat tissue around the HA disc. The HA disks fixed in formalin were decalcified in K-CX solution (Falma Co., Tokyo, Japan) and embedded in paraffin. They were cut parallel down the centre of specimens to the round surface of the HA discs and stained with hematoxylin and eosin (H-E).

\subsection{RNA Isolation and Real-Time Quantitative PCR}

We measured the gene expression levels of ALP and OC to confirm osteogenesis in the harvested disks. RNA was isolated from four disks from each group using an Isogen RNA extraction kit (Nippon Gene Co. Ltd., Toyama, Japan). Briefly, each sample was placed in Isogen solution containing matrix beads and disrupted with a FastPrep FP24 Cell Disrupter (Qbiogene, Inc., Carlsbad, CA, USA) $[12,17]$. Subsequently, the remaining steps of RNA isolation were performed according to the manufacturer's instructions. To measure mRNA expression levels, we conducted real-time quantitative PCR (Applied Biosystems StepOnePlus; Applied Biosystems, Norwalk, CT, USA), using primers for ALP, OC and glyceraldehyde-3-phosphate dehydrogenase (GAPDH, internal standard) for rat cDNAs as previously described [22]. Target ALP and OC mRNA levels were compared after correcting to GAPDH mRNA levels as an internal standard, to adjust for differences in the efficiency of reverse transcription between samples. ALP (Rn00564931 m1), OC (Rn $01455285 \mathrm{~g} 1$ ) and GAPDH (Rn99999916 s1) primer and probe sets were purchased from Applied Biosystems (Foster City, CA, USA). Thermal cycle conditions were $20 \mathrm{sec}$ at $95^{\circ} \mathrm{C}$ for activation of TaqMan Fast Universal PCR Master Mix, followed by 40 cycles of $1 \mathrm{sec}$ at $95^{\circ} \mathrm{C}$ for denaturing and $20 \mathrm{sec}$ at $60^{\circ} \mathrm{C}$ for annealing and extension. This experiment was performed in duplicate. 


\subsection{Statistics}

Statistical significant was determined by one-way ANOVA post-hoc multiple comparisons using Tukey's test. A $p$ value of 0.05 was considered statistically significant.

\section{RESULTS}

\subsection{Osteogenic Potential of Serial Passaged Cells in Vitro}

Figure 1 shows ALP (Figure 1(a)) and Alizarin Red $S$ staining (Figure 1(b)) of BMSCs that were cultured with or without osteogenic-induced medium at P1, P2 and P3. P1 cells cultured with osteogenic-induced medium showed strong positive staining for ALP and Alizarin Red S, whereas P3 cells showed faint staining for ALP and no Alizarin Red S staining. ALP staining in P2 cells seems weaker than in P1 cells and the size of mineralized nodule in P2 cells appears smaller than P1 cells. Progressively weaker staining was seen in P1, P2 and P3 cells, indicating that osteogenic potential of seriallypassaged BMSCs is lost by P3. P1, P2 and P3 cells cultured without osteogenic-induced medium were negative for ALP and Alizarin Red S staining.

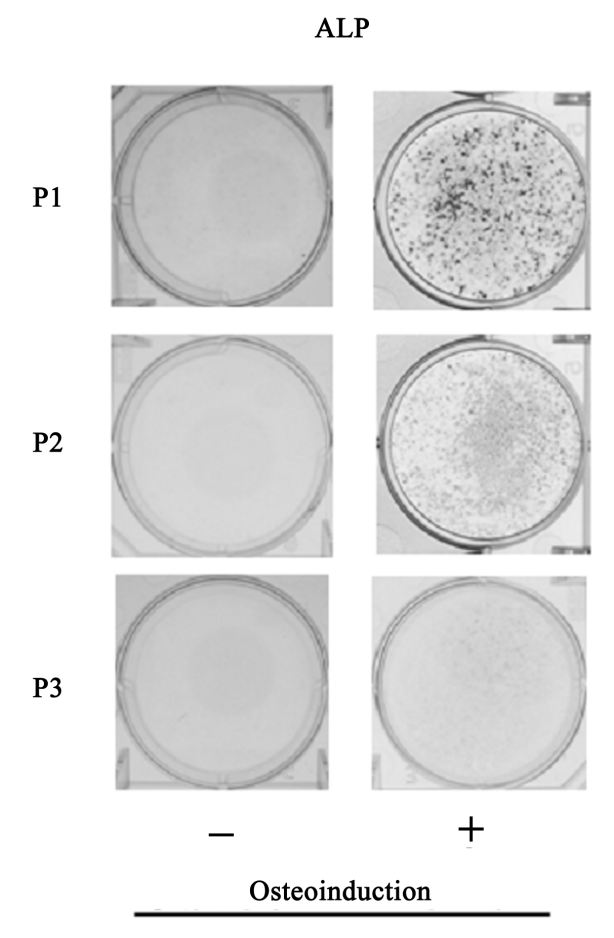

ALP

(a)
Figure 2 shows ALP activity and calcium deposition, which were standardized to DNA content in each group. The activity of P2 cells cultured in osteogenic-induced medium were significantly lower than that of the positive control, indicating that passaging of cells decreases the osteoblastic phenotype of cultured cells (Figure 2(a)). There was a significant difference in calcium deposition between the positive control and P2 cells, indicating loss of mineralization ability with increasing passage (Figure 2(b)).

\subsection{Bone Ormation of P3-Cell/HA Constructs after Implantation}

Figure 3 shows representative histological sections of the harvested HA constructs 4 weeks after implantation. Little bone formation occurred in the pores of the control group (the P3 cell/HA constructs: Figure 3(a)) and the small loading group (P3 cell/HA constructs with $3.5 \mathrm{~cm}$ dish P3 cell-loading: Figure 3(b)). In contrast, abundant bone formation, including with osteoblasts and osteocytes, was observed in the large loading group (P3 cell/HA constructs with $10 \mathrm{~cm}$ dish P3 cell-loading: Figure 3(c)).
Alizarin Red S

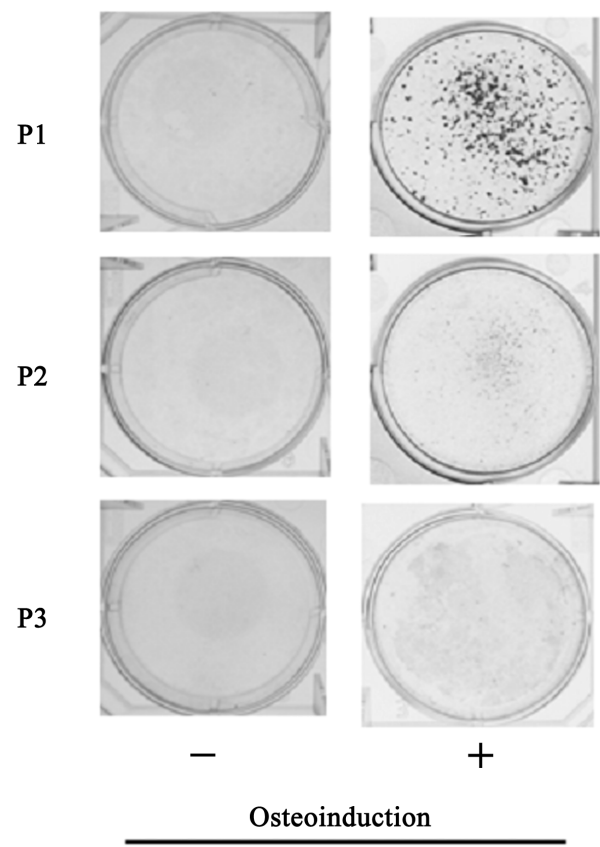

(b)

Figure 1. ALP (a) and Alizarin Red S staining (b). P1, P2 and P3 cells were cultured in 6-well plates for 14 days. Positive staining for ALP and Alizarin Red S was observed in P1 cells cultured with osteogenic-induced medium. Progressively weaker staining was seen in P1, P2 and P3 cells cultured with osteogenic-induced medium. By contrast, P1, P2 and P3 cells cultured without osteogenic-induced medium were negative for ALP and Alizarin Red S staining. 


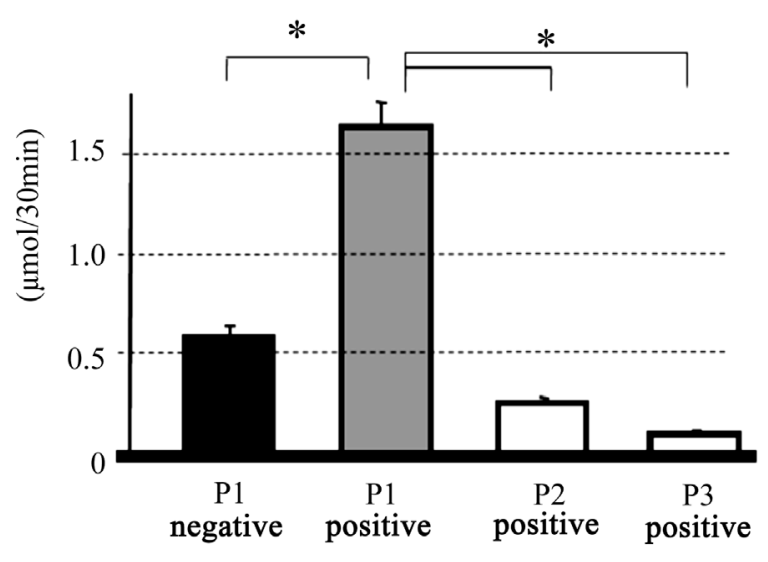

(a)

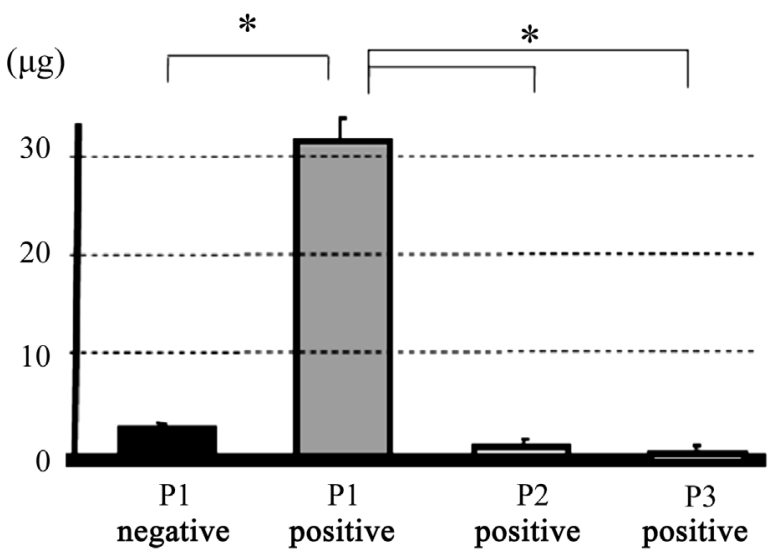

(b)

Figure 2. ALP activity (a) and calcium deposition (b), standardized to DNA content. P1 and P2 represent the passage of cells. Positive and negative indicate cells cultured with or without osteogenic-induced medium, respectively. Asterisk indicates $p<0.05$.

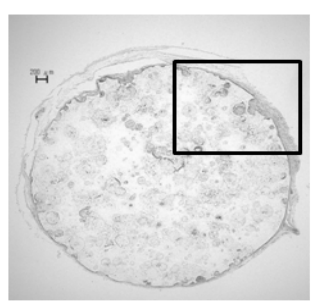

(a)

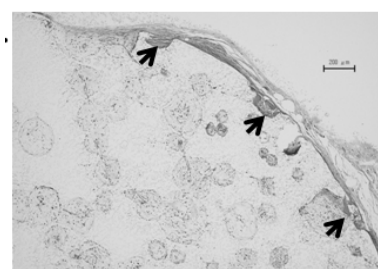

(d)

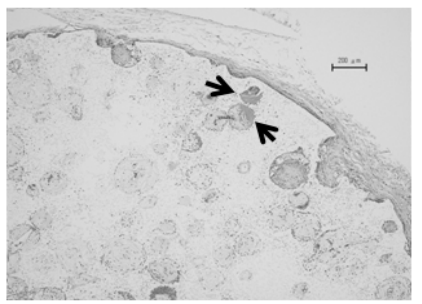

(b)

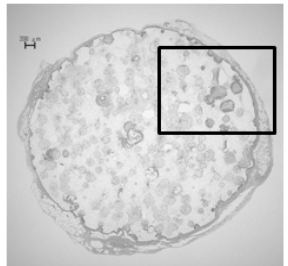

(e)

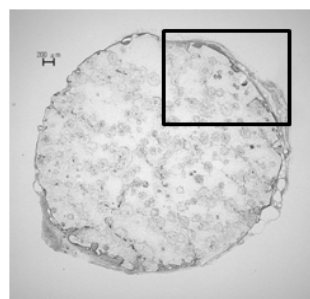

(c)

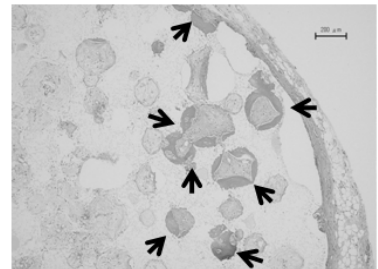

(f)

Figure 3. Representative histological sections of the harvested HA constructs 4 weeks after implantation. Little bone formation was observed in the pore of the control group (the P3 cell/HA construct: Figures 3(a) and (b)) and small cell-loading group (the P3 cell/HA construct with $3.5 \mathrm{~cm}$ dish cell-loading: Figures 3(c) and (d)). In contrast, abundant bone formation together with osteoblasts and osteocytes was seen in large cell-loading group (the P3 cell/HA construct with $10 \mathrm{~cm}$ dish cell-loading: Figures 3(e) and (f)). Arrows indicate bone tissue. Bar $=200 \mu \mathrm{m}$.

Figure 4 shows ALP and OC mRNA expression levels evaluated by real-time PCR. The ALP expression in the large loading group was significantly higher than in the control and small loading groups (Figure 4(a)). OC expression levels were similar to ALP levels (Figure 4(b)).

\section{DISCUSSION}

The present study shows that serial passaging of BMSCs decreases the osteogenic potential in vitro. Consequently, little bone formation was seen in the pore of the construct when P3 cells were combined with HA disk (P3 cell/HA construct) and implanted into a subcutane- ous site. However, when additional P3 cells were loaded into the P3 cell/HA construct and implanted, obvious bone formation was seen, indicating that the osteogenic potential of the construct is regained by additional loading of cells cultured in medium with Dex and ascorbic acid. We assume that the additional cell-loading, using the cell sheet culture method with short centrifugation, is more effective at loading cells into the construct than the conventional cell suspension method. Cell suspensions of high cell density can be easily prepared, however, it is difficult to achieve high loading of cells into ceramics. In the present study, we prepared $\mathrm{P} 3$ cells using a $3.5 \mathrm{~cm}$ 


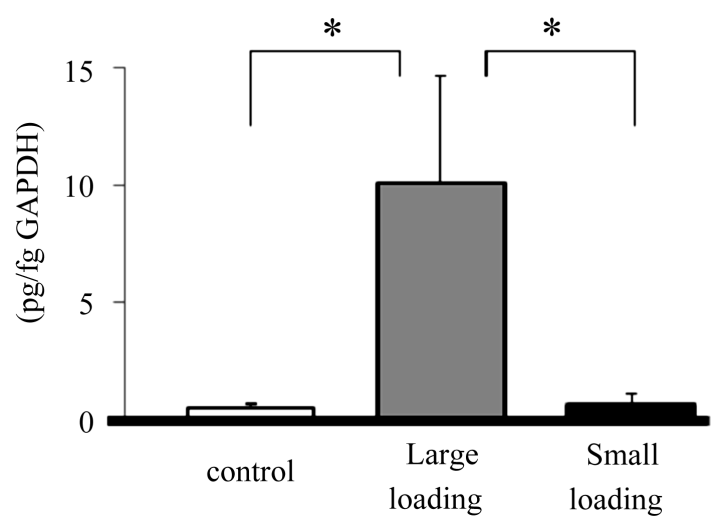

(a)

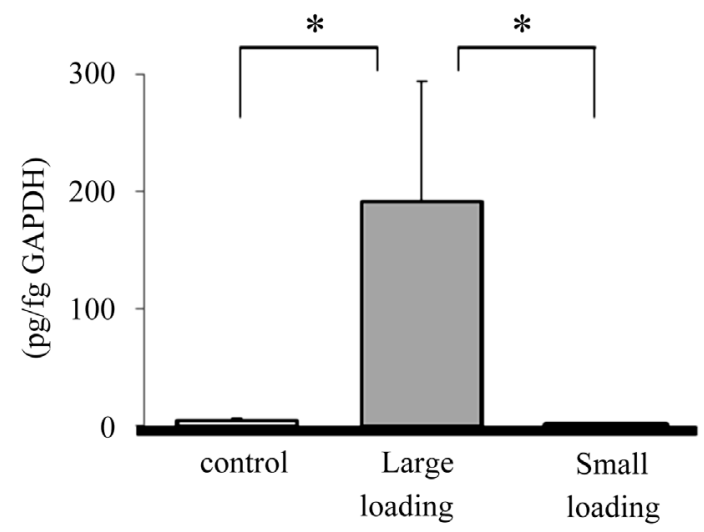

(b)

Figure 4. Real-time PCR of ALP and OC mRNA expression. OC expression in the large loading group was significantly higher than those in the control and small loading groups (Figure 4(a)). The tendency of ALP expression (Figure 4(b)) was similar to OC expression. Control: control group, Large loading: large cell-loading group, Small loading: small cell-loading group. Asterisk indicates $p<0.05$.

and $10 \mathrm{~cm}$ culture dishes. The culture area of $10 \mathrm{~cm}$ dish is approximately 5 times larger than the $3.5 \mathrm{~cm}$ dish. Consequently, the cell density is also 5 times more in a $10 \mathrm{~cm}$ dish. The construct with the $3.5 \mathrm{~cm}$ dish P3 cell-loading showed smaller bone formation 4 weeks after implantation compared with the $10 \mathrm{~cm}$ dish P3 cell-loading constructs, indicating that the loaded cell number could be an important factor to form bone tissue in the construct combined with serially-passaged cells.

Seeded cells may contain a combination of differentiated and undifferentiated cells after passaging. Differentiated cells have a limited capacity for proliferation $[23,24]$ and as the cells proliferating from the seeded cells are undifferentiated cells, there is a decrease in the percentage of osteogenic cells in the culture $[8,25]$. Bone marrow cells are a mixed population of cells, including BMSCs, fibroblasts and endothelial cells. After serial passaging, the population of cultured cells changes. Therefore, we assume that percentage of osteogenic cells might decrease during cell culture and result in less ALP activity and mineralization by $\mathrm{P} 2$ and $\mathrm{P} 3$ passages.

Kumar et al. [26] have reported a method for the preparation of cell sheet/HA constructs using a thermosensitive polymer to fabricate the cell sheet. In their report, they showed rapid and complete cellularization to HA by wrapping it with a cell sheet fabricated from a human osteosarcoma cell line. Thus, we think that it is possible to load a lot of cells onto the construct by using the wrapping method with $\mathrm{P} 1$ cell sheets. The purpose of the present study was to evaluate the osteogenic potential of serially-passaged cells. We used P3 cells to assess the osteogenic potential of $\mathrm{P} 3$ cell/HA constructs. At P3, cell culture with the cell sheet preparation method formed incomplete sheet like fragments. Therefore, we used centrifugation to load the incomplete cell sheet onto the constructs as an additional cell-loading technique. Because little bone formation was seen in the harvested construct with $3.5 \mathrm{~cm}$ dish cell-loading, the amount of bone formation could depend on the loading cell number on the HA. The decreased number of osteogenic cells could be compensated by the additional cell-loading from a $10 \mathrm{~cm}$ culture dish.

Recently, tissue engineering has been applied clinically $[19,20]$, with some of the technology using cell culture. Bone marrow cells, including BMSCs, can be obtained from patients by needle aspiration and thus the invasiveness is much less compared with harvesting the patient's bone. Therefore, bone marrow cells containing BMSCs are considered a good candidate for tissue-engineered bone. In general, BMSCs need to be expanded by serial passaging to obtain enough cells for their clinical application in tissue engineering. However, the osteogenic potential of cultured BMSCs would be lost after serial passaging. Brugge et al. [8] reported that ALP activity and calcium content in cultured cells were not detected at $\mathrm{P} 3$ in vitro. They also reported that to maintain the osteogenic potential of cells during subcultures requires the addition of Dex to the culture medium as an osteoblastic differentiation factor. However, BMSCs lose their osteogenic potential after several passages even when Dex was added continuously. They concluded that the serial passaging of BMSCs results in the loss of osteogenic potential. A limitation of their report was that it only contained experimental results of an in vitro study. Therefore, we decided to examine the osteogenic potential of serially-passaged BMSCs and whether they can differentiate into osteoblasts and subsequently promote bone formation after in vivo transplantation. We evaluated the osteogenic potential of P1-P3 cells in this study as lower-passage BMSCs are generally used in clinical 
applications for tissue-engineered bone reconstruction.

There are a number of limitations of the study that should be acknowledged. First is the use of rat BMSCs in the present study. For the clinical application of this technique, human BMSCs should be used to determine whether our cell-loading method can restore the oseteogenic potential of HA constructs combined with serially-passaged cells. Second, the culture technique for the preparation of cell sheets formed incomplete sheets at P3. Therefore, further improvement of cell sheet preparation for serially-passaged cells is needed. A complete cell sheet created by serial passaging could load large amounts of cells onto the constructs without centrifugation and resulted in increased bone formation. Third, we used only HA in the present study. Other ceramics, such as $\beta$-tricalcium phosphate ( $\beta$-TCP), are also commonly used clinically [27-29]. Therefore, other types of ceramics should be tested to confirm our results.

\section{CONCLUSION}

The present study indicated that osteogenic potential of BMSCs decreases by serial passaging. However, bone formation could be regained using the additional cellloading technique. Owing to its usage of serially-passaged cells, this method could be applied in cases of hard tissue reconstruction, which requires the serial passaging of BMSCs to obtain a suitable number of cells. This technique, along with the cell suspension method, can be used to prepare cell-loaded constructs from ceramics, such as HA and $\beta$-TCP.

\section{ACKNOWLEDGEMENTS}

We thank Ms M. Yoshimura and Ms M. Matsumura (Nara Medical University School of Medicine, Japan) for their technical assistance. This study was supported by Takeda Science Foundation.

\section{REFERENCES}

[1] Owen, M. (1988) Marrow stromal stem cells. Journal of Cell Science, 10, 63-76.

[2] Ohgushi, H. and Caplan, A.I. (1999) Stem cell technology and bioceramics: From cell to gene engineering. Journal of Biomedical Materials Research, 48, 913-927. doi:10.1002/(SICI)1097-4636(1999)48:6<913::AID-JBM 22>3.0.CO;2-0

[3] Ohgushi, H., Yoshikawa, T., Nakajima, H., Tamai, S., Dohi, Y. and Okunaga, K. (1999) $\mathrm{Al}_{2} \mathrm{O}_{3}$ doped apatitewollastonite containing glass ceramic provokes osteogenic differentiation of marrow stromal stem cells. Journal of Biomedical Materials Research, 44, 381-388. doi:10.1002/(SICI)1097-4636(19990315)44:4<381::AIDJBM3>3.0.CO;2-E

[4] Sonal, R., Jackson, J.D., Brusnahan, S.K., O'Kane, B. J. and Sharp, J.G. (2012) Characterization of a mesenchy- mal stem cell line that differentiates to bone and provides niches supporting mouse and human hematopoietic stem cells. Stem Cell Discovery, 2, 5-14. doi:10.4236/scd.2012.21002

[5] Brazelton, T.R., Rossi, F.M., Keshet, G.I. and Blau, H.M. (2000) From marrow to brain: Expression of neuronal phenotypes in adult mice. Science, 290, 1775-1779. doi:10.1126/science.290.5497.1775

[6] Jiang, Y., Jahagirdar, B.N., Reinhardt, R.L., Schwartz, R.E., Keene, C.D., Ortiz-Gonzalez, X.R., Reyes, M., Lenvik, T., Lund, T., Blackstad, M., Du, J., Aldrich, S., Lisberg, A., Low, W.C., Largaespada, D.A. and Verfaillie, C.M. (2002) Pluripotency of mesenchymal stem cells derived from adult marrow. Nature, 418, 41-49. doi:10.1038/nature00870

[7] Krause, D.S. (2002) Plasticity of marrow-derived stem cells. Gene Therapy, $\mathbf{9}, 754-758$. doi:10.1038/sj.gt.3301760

[8] Ter Brugge, P.J. and Jansen, J.A. (2002) In vitro osteogenic differentiation of rat bone marrow cells subcultured with and without dexamethasone. Tissue Engineering, 8, 321-331. doi:10.1089/107632702753725076

[9] Matsushima, A., Kotobuki, N., Tadokoro, M., Kawate, K., Yajima, H., Takakura, Y. and Ohgushi, H. (2009) In vivo osteogenic capability of human mesenchymal cells cultured on hydroxyapatite and on beta-tricalcium phosphate. Artificial Organs, 33, 474-481. doi:10.1111/j.1525-1594.2009.00749.x

[10] Akahane, M., Shigematsu, H., Tadokoro, M., Ueha, T., Matsumoto, T., Tohma, Y., Kido, A., Imamura, T. and Tanaka, Y. (2010) Scaffold-free cell sheet injection results in bone formation. Journal of Tissue Engineering and Regenerative Medicine, 4, 404-411. doi:10.1002/term.259

[11] Nakamura, A., Akahane, M., Shigematsu, H., Tadokoro, M., Morita, Y., Ohgushi, H., Dohi, Y., Imamura, T. and Tanaka, Y. (2010) Cell sheet transplantation of cultured mesenchymal stem cells enhances bone formation in a rat nonunion model. Bone, 46, 418-424. doi:10.1016/j.bone.2009.08.048

[12] Nakamura, A., Dohi, Y., Akahane, M., Ohgushi, H., Nakajima, H., Funaoka, H. and Takakura, Y. (2009) Osteocalcin secretion as an early marker of in vitro osteogenic differentiation of rat mesenchymal stem cells. Tissue Engineering Part C: Methods, 15, 169-180. doi:10.1089/ten.tec.2007.0334

[13] Akahane, M., Ohgushi, H., Yoshikawa, T., Sempuku, T., Tamai, S., Tabata, S. and Dohi, Y. (1999) Osteogenic phenotype expression of allogeneic rat marrow cells in porous hydroxyapatite ceramics. Journal of Bone and Mineral Research, 14, 561-568. doi:10.1359/jbmr.1999.14.4.561

[14] Bianco, P. and Robey, P.G. (2001) Stem cells in tissue engineering. Nature, 414, 118-121. doi: $10.1038 / 35102181$

[15] Dong, J., Kojima, H., Uemura, T., Kikuchi, M., Tateishi, T. and Tanaka, J. (2001) In vivo evaluation of a novel porous hydroxyapatite to sustain osteogenesis of transplanted bone marrow-derived osteoblastic cells. Journal of Biomedical Materials Research, 57, 208-216. 
doi:10.1002/1097-4636(200111)57:2<208::AID-JBM116 0>3.0.CO;2-N

[16] Petite, H., Viateau, V., Bensaid, W., Meunier, A., de Pollak, C., Bourguignon, M., Oudina, K., Sedel, L. and Guillemin, G. (2000) Tissue-engineered bone regeneration. Nature Biotechnology, 18, 959-963. doi:10.1038/79449

[17] Shigematsu, H., Akahane, M., Dohi, Y., Nakamura, A., Ohgushi, H., Imamura, T. and Tanaka, Y. (2010) Osteogenic potential and histological characteristics of mesenchymal stem cell sheet/hydroxyapatite constructs. The Open Tissue Engineering and Regenerative Medicine Journal, 2, 63-70. doi:10.2174/1875043500902010063

[18] Akahane, M., Nakamura, A., Ohgushi, H., Shigematsu, H., Dohi, Y. and Takakura, Y. (2008) Osteogenic matrix sheet-cell transplantation using osteoblastic cell sheet resulted in bone formation without scaffold at an ectopic site. Journal of Tissue Engineering and Regenerative Medicine, 2, 196-201. doi:10.1002/term.81

[19] Wakitani, S., Imoto, K., Yamamoto, T., Saito, M., Murata, N. and Yoneda, M. (2002) Human autologous culture expanded bone marrow mesenchymal cell transplantation for repair of cartilage defects in osteoarthritic knees. Osteoarthritis and Cartilage, 10, 199-206. doi:10.1053/joca.2001.0504

[20] Ohgushi, H., Kotobuki, N., Funaoka, H., Machida, H., Hirose, M., Tanaka, Y. and Takakura, Y. (2005) Tissue engineered ceramic artificial joint $-E x$ vivo osteogenic differentiation of patient mesenchymal cells on total ankle joints for treatment of osteoarthritis. Biomaterials, 26, 4654-4661. doi:10.1016/j.biomaterials.2004.11.055

[21] Kawate, K., Yajima, H., Ohgushi, H., Kotobuki, N., Sugimoto, K., Ohmura, T., Kobata, Y., Shigematsu, K., Kawamura, K., Tamai, K. and Takakura, Y. (2006) Tissue-engineered approach for the treatment of steroidinduced osteonecrosis of the femoral head: Transplantation of autologous mesenchymal stem cells cultured with beta-tricalcium phosphate ceramics and free vascularized fibula. Artifical Organs, 30, 960-962. doi:10.1111/j.1525-1594.2006.00333.x

[22] Akahane, M.T.U., Shimizu, T., Shigematsu, H., Kido, A., Omokawa, S., Kawate, K., Imamura, T. and Y. Tanaka. (2010) Cell Sheet Injection as a technique of osteogenic supply. International Journal of Stem Cells, 3, 138-143.

[23] McCulloch, C.A., Strugurescu, M., Hughes, F., Melcher, A.H. and Aubin, J.E. (1991) Osteogenic progenitor cells in rat bone marrow stromal populations exhibit self-renewal in culture. Blood, 77, 1906-1911.

[24] Aubin, J.E. (1998) Advances in the osteoblast lineage. Biochemistry and Cell Biology, 76, 899-910. doi:10.1139/099-005

[25] Kadiyala, S., Young, R.G., Thiede, M.A. and Bruder, S.P. (1997) Culture expanded canine mesenchymal stem cells possess osteochondrogenic potential in vivo and in vitro. Cell Transplantation, 6, 125-134. doi:10.1016/S0963-6897(96)00279-5

[26] Anil, K.P.R., Varma, H.K. and Kumary, T.V. (2005) Rapid and complete cellularization of hydroxyapatite for bone tissue engineering. Acta Biomaterialia, 1, 545-552. doi:10.1016/j.actbio.2005.05.002

[27] Ogose, A., Hotta, T., Hatano, H., Kawashima, H., Tokunaga, K., Endo, N. and Umezu, H. (2002) Histological examination of beta-tricalcium phosphate graft in human femur. Journal of Biomedical Materials Research, 63, 601-604. doi:10.1002/jbm.10380

[28] Yamamoto, T., Onga, T., Marui, T. and Mizuno, K. (2000) Use of hydroxyapatite to fill cavities after excision of benign bone tumours. Clinical results. Journal of Bone \& Joint Surgery, British Volume, 82, 1117-1120. doi:10.1302/0301-620X.82B8.11194

[29] Schindler, O.S., Cannon, S.R., Briggs, T.W. and Blunn, G.W. (2008) Composite ceramic bone graft substitute in the treatment of locally aggressive benign bone tumours. Journal of Orthopaedic Surgery (Hong Kong), 16, 66-74. 